\title{
SFR bulge-to-disk ratios from the CALIFA IFS nearby galaxies survey
}

\author{
C. Catalán-Torrecilla ${ }^{1}$, A. Gil de Paz ${ }^{1}$, A. Castillo-Morales ${ }^{1}$, J. \\ Méndez-Abreu ${ }^{2}$, J. Iglesias-Páramo ${ }^{3}$, B.F. Madore ${ }^{4}$, S. Pascual ${ }^{1}$, T. \\ Ruiz-Lara $^{5}$, A. de Lorenzo-Cáceres ${ }^{2}$, L. Sánchez-Menguiano ${ }^{5}$, S. \\ Sánchez ${ }^{6}$ and CALIFA Collaboration \\ ${ }^{1}$ Departamento de Astrofísica y CC. de la Atmósfera, Universidad Complutense de Madrid, \\ E-28040, Madrid, Spain ; email: ccatalan@ucm.es \\ ${ }^{2}$ School of Physics and Astronomy, University of St. Andrews, KY16 9SS St. Andrews, UK \\ ${ }^{3}$ Instituto de Astrofísica de Andalucía-CSIC, Glorieta de la Astronomía,18008 Granada, Spain \\ ${ }^{4}$ Observatories of the Carnegie Institution of Washington, Pasadena, CA 91101 \\ ${ }^{5}$ Dpto. de Física Teórica y del Cosmos, Universidad de Granada, E-18071 Granada, Spain \\ ${ }^{6}$ Instituto de Astronomía,Universidad Nacional Autonóma de México, 04510, México D.F.
}

\begin{abstract}
Our aim is to study the Star Formation Rate (SFR) by galaxy components such as bulges, bars and disks in a representative sample of nearby galaxies. A 2-dimensional (2D) photometric decomposition approach (GASP2D) is used to obtain these components. The availability of IFS data for the CALIFA galaxies makes possible to go one step further as we can apply the previous decompositions over 3D datacubes to disentangle the spatial distribution of the SFR over different components free from the limitations associated to narrow-band imaging.
\end{abstract}

Keywords. galaxies: evolution, SF, structure, techniques: photometric, spectroscopic

\section{Introduction}

Understanding the evolution of the SFR density by galaxy components (bulge, bar and disk) will shed some light on the models of galaxy formation and evolution. Our ultimate goal is to answer: how is the local SFR density spatially distributed over galaxies and how this has evolved with redshift and as a function of the galaxies' global properties?

\section{Method and Results}

We study the spatial distribution of the SFR using extincion-corrected $\mathrm{H} \alpha$ luminosity in a sample of 83 nearby galaxies from the CALIFA IFS survey. Prior to this study, we have verified that we can recover the integrated attenuation-corrected $\mathrm{H} \alpha \mathrm{SFR}$ in a sample of 272 galaxies making use of single-band and hybrid tracers (C. Catalán-Torrecilla et al.(2015)).

To obtain the SFR in the bulge, in the bar and in the disk separately, we perform a $2 \mathrm{D}$ photometric decomposition using the algorithm GASP2D (Méndez-Abreu et al.(2008)) with SDSS $g$-band images. As a result, we determine the light coming from each component as the ratio between the luminosity in every component (bulge, bar or disk) and the total luminosity of the galaxy. Then, for each galaxy we multiply the IFS datacubes by the corresponding weight maps to recover the luminosity in each component (see left and central panels in Fig. 1). Finally, we derive the spectrum associated to each galaxy component integrating the spatial information in the weighted datacube using an elliptical aperture covering the whole galaxy. To get the $\mathrm{H} \alpha$ and $\mathrm{H} \beta$ fluxes, we subtract the stellar continuum by fitting a combination of SSP evolutionary models (Fig. 1).

Finally, we obtain the percentage of the total $\operatorname{SFR}(\mathrm{H} \alpha)$ that is concentrated in the bulges, the bars and the disks for different morphological types (Fig. 2). There is a clear decrease in the values of the SFR in the bulge as we go to the latest spiral galaxies while the values of the SFR in the disk increase, except for the case of Sb-Sbc where the presence of the bar is reducing the $\mathrm{SFR}$ in the disk in favor of the SFR in the bar. 

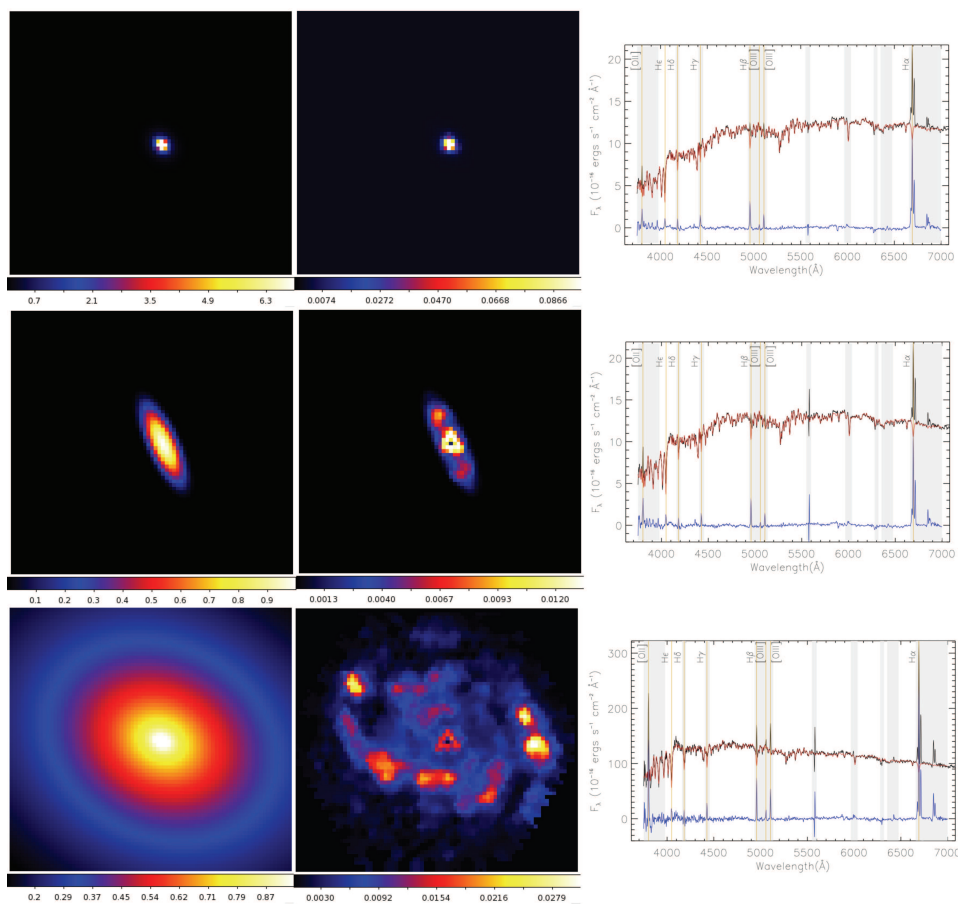

Figure 1. Left panels: model for the galaxy NGC5947 derived from a 2D photometric decomposition (GASP2D) fitting the bulge, bar and disk components. Central panels: maps showing the distribution of the continuum subtracted $\mathrm{H} \alpha$ luminosity for each component. Right panels: integrated spectra for the bulge, the bar and the disk. Black is the original spectrum, red is the best model to fit the stellar population and blue is the pure emission-line spectrum.

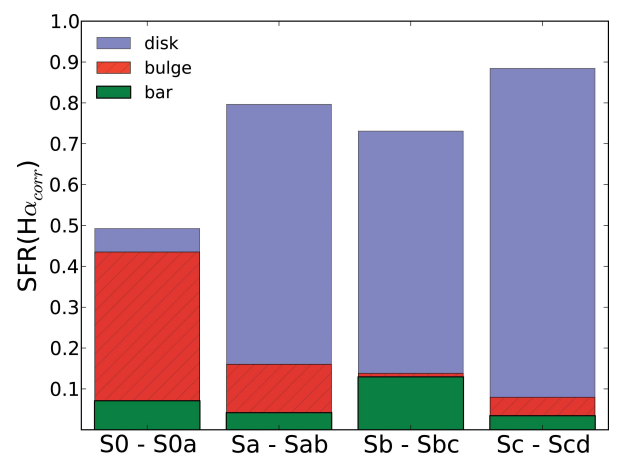

Figure 2. Distribution of the attenuation-corrected $\operatorname{SFR}(\mathrm{H} \alpha)$ by galaxy components (bulge, bar and disk) with the morphological type. The values shown in this plot have been normalized to the total number of galaxies available in each bin.

\section{References}

Catalán-Torrecilla C., Gil de Paz A., Castillo-Morales A. et al. 2015, ArXiv:1507.03801

Méndez-Abreu, J., Aguerri, J. A. L. , Corsini, E. M. and Simonneau, E., 2008, A\&A 478, 353 